\title{
Internal Stability of Dynamically Quantised Control for Stochastic Scalar Plants *
}

\author{
Assaf Gurt* Girish N. Nair* \\ * Department of Electrical and Electronic Engineering, University of \\ Melbourne, VIC 3010, Australia \\ E-mail:\{a.gurt@ee.unimelb.edu.au,gnair@unimelb.edu.au\}
}

\begin{abstract}
In this paper we study conditions under which an unstable stochastic scalar linear plant with unbounded noise can be internally stabilised using 'zooming'-like coding and control schemes having dynamic, finite-dimensional internal states. Such structures are known to be needed in communicationconstrained control when no bound on the plant noise is available. However, previous schemes were based on coders and controllers starting with identical internal states. In this paper, we remove this assumption and explicitly construct a finite-dimensional coding and control policy that yields mean square stability of all state variables, for a random initial plant state and arbitrary initial encoder and controller states. This holds for any bit rate down to the universal minimum of the Data Rate Theorem. Furthermore, we show that despite the unbounded noise, the error and proportional errors between the scaling factors of the encoder and controller tend to zero in mean square and almost sure senses respectively. This suggests that the policy will still maintain mean square internal stability in the presence of channel bit errors, provided the bit error rate is sufficiently low. We support these conclusions with simulations.
\end{abstract}

Keywords: Control under communication constraints, stochastic control

\section{INTRODUCTION}

Due to the rapid growth in communication technology over the last few years, it is becoming increasingly common to employ digital finite capacity channels for the exchange of information between many control system components such as sensors and actuators. Such applications arise in the broad areas of sensor networks and industrial and defence systems. Although the total capacity of the network may be large, each component may be allocated only a small portion, introducing limitations on the achievability of the control objective. As a consequence, many papers have appeared recently in the literature which addressed the problem of controlling an unstable plant using quantised control schemes with limited bit rate; see Nair et al. (2007) for an overview.

For unstable linear time-invariant (LTI) plants with no or bounded noise, it was proven in Wong and Brockett (1999); Baillieul (2001); Tatikonda and Mitter (2004) that memoryless quantisers and controllers suffice to ensure boundedness of states at any bit rate down to a universal minimum. However, in Brockett and Liberzon (2000); Liberzon (2003), it was shown that by employing a 'zooming' scheme featuring a real-valued quantiser range that is dynamically adjusted according to the most recent quantiser output, the state of a noiseless linear plant could be asymptotically stabilised to the origin; this was an impossible feat with static coding and control laws and provided the initial motivation for dynamically quantised control. In Nair and Evans (2004), another dynamic quantisation scheme was subsequently proposed and shown to mean-square-stabilise the

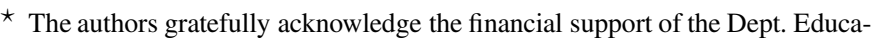
tion, Science and Training, the Australian Research Council and National ICT Australia Ltd.
}

states of a stochastic LTI plant with unbounded process and measurement noise, at any rate down the the universal minimum. It was also proven there that with unbounded process noise and strictly unstable plant dynamics, stochastic stability in any sense was impossible with memoryless or bounded quantisation, regardless of the number of quantiser points.

From a more general point of view, the dynamically quantised schemes mentioned above are examples of of finitedimensional encoders and controllers that have real-valued internal states. The crucial assumption on which their analyses rested was that the initial states of the encoder and controller were identical. In the absence of channel bit errors, this enabled the encoder to accurately track how the controller state evolved with each transmitted bit. Given the unstable dynamics of the plant and the inherently discontinuous nature of quantisation, it was unclear if the schemes proposed above had any stability margin with respect to initial internal state errors.

This is a matter of concern in practice, since even with channel error correction coding, the probability of bit error will never be exactly zero. With arbitrarily high probability, a bit will be detected erroneously after a sufficiently long time, at which point the controller internal state will differ from what the encoder predicts.

In this paper, we present a preliminary study of and partial solution to this problem, for the case of an unstable scalar LTI plant with unbounded process and measurement noise with 'fat' distribution tails. We formulate the question in section 2 and construct in section 3 a finite-dimensional coding and control scheme. In section 4 , we then prove that it achieves mean square stability of all closed-loop state variables, under a sufficient condition on the channel bit rate. By introducing a periodically 
time-varying scheme of sufficiently long coding cycle length, this rate condition approaches the minimum rate expression of Nair and Evans (2004).

In section 4, we also discuss the fact that if mean square stability is assured, then the internal states of the encoder and controller must reach asymptotic agreement in mean square and proportional almost-sure senses. This suggests that, without requiring any channel bit error correction coding, the scheme may also be able to achieve mean square stability over erroneous channels, provided that the bit error rate is much lower than the rate at which agreement is reached. Simulation results supporting these conclusions are presented and discussed in sec. 5 .

Although we focus here on scalar plants, similar results can be shown to hold with vector-valued plant states by transforming into real Jordan form [Horn and Johnson (1985)] and applying a variant of the construction here to each decoupled plant mode.

\section{FORMULATION}

Consider the stochastic, discrete-time, linear time-invariant (LTI) plant:

$$
\begin{aligned}
X(t+1) & =a X(t)+b U(t)+V(t), \\
Y(t) & =c X(t)+W(t),
\end{aligned}
$$

where $X(t), U(t), Y(t), V(t), W(t)$ are the plant state, input, output, process noise and measurement noise respectively. We assume that, for some $\varepsilon>0$, the initial state has a finite $(2+\varepsilon)$ th moment $\mathrm{E}\left\{|X(0)|^{2+\varepsilon}\right\}<\infty$ and that the process and observation noise have uniformly bounded $(2+\varepsilon)$-th moments: $\sup _{t \in \mathbb{Z}_{\geq 0}} \mathrm{E}\left\{|V(t)|^{2+\varepsilon}\right\}, \sup _{t \in \mathbb{Z}_{\geq 0}} \mathrm{E}\left\{|W(t)|^{2+\varepsilon}\right\}<\infty$. Such moment conditions are common in quantisation theory [Graf and Luschgy (2000)] and admit not just exponentially-decaying distributions such as the Gaussian, but also distributions with fat tails that diminish according to a power law.

Suppose that the plant above is controlled by means of a feedback signal transmitted over a digital channel from the output sensor to a controller that generates regulating inputs $U(0), U(1), \ldots$. Specifically, at time $t$ a discrete-valued symbol $S(t)$ belonging to a time-varying, finite alphabet $S(t)$ is transmitted by an encoder onto the channel. We define the data rate of the channel to be

$$
R:=\liminf _{\tau \rightarrow \infty} \frac{1}{\tau} \sum_{t=0}^{\tau-1} \log _{2}|\mathcal{S}(t)| \text { (bits/sample) }
$$

and assume here that $R<\infty$; this constrains the rate at which information can be signalled from sensor to controller.

To minimise the computational burden, we wish to restrict to coding and control laws that can be implemented in finitedimensional form. That is, for some $c \in \mathbb{N}$ and any time $t$, we are interested in controllers having a dynamic, finite-dimensional internal state $\Psi^{\mathrm{c}}(t) \in \mathbb{R}^{c}$ which evolves in a possibly nonlinear way depending on the past state and the latest received symbol, and such that the control signal is a (possibly nonlinear) function of the current internal state:

$$
\left(\Psi^{\mathrm{c}}(t-1), S(t-1)\right) \mapsto \Psi^{\mathrm{c}}(t), \quad \Psi^{\mathrm{c}}(t) \mapsto U(t) \in \mathbb{R} .
$$

Similarly, for some $e \in \mathbb{N}$ and $\forall t \in \mathbb{Z}_{>0}$, the encoder has another internal state $\Psi^{\mathrm{e}}(t) \in \mathbb{R}^{e}$ which evolves as

$$
\left(\Psi^{\mathrm{e}}(t-1), Y(t)\right) \mapsto \Psi^{\mathrm{e}}(t), \quad \Psi^{\mathrm{e}}(t) \mapsto S(t) \in \mathcal{S}(t) .
$$

We define a finite-dimensional encoder-controller (FDEC) by the coding alphabet sequence $\{S(t)\}_{t=0}^{\infty}$ together with the finitedimensional and possibly time-varying mappings (4)-(5).

Many of the of the quantised estimation or control schemes studied in the literature Wong and Brockett (1999); Brockett and Liberzon (2000); Liberzon (2003) can be viewed as specific examples of FDEC's. In Nair and Evans (2004), a FDEC was constructed that yielded mean square stability (MSS) of a stochastic LTI plant with an $n$-dimensional state vector for any rate (3) that satisfied

$$
R>\sum_{i=1}^{n} \max \left\{\log _{2}\left|\lambda_{i}\right|, 0\right\},
$$

where $\lambda_{1}, \ldots \lambda_{n}$ were the eigenvalues of the open-loop plant dynamic matrix. As this was also known to be a necessary condition for plant mean square stabilisability, the construction in Nair and Evans (2004) thus achieved plant MSS at any specified bit rate down to the lowest possible.

However, a crucial assumption behind the analyses of all these aforementioned schemes was that the initial internal states $\Psi^{\mathrm{e}}(0), \Psi^{\mathrm{c}}(0)$ be identical. If no channel errors occured, this enabled the encoder to mimic the controller internal states exactly, by running a local version of the controller's update and output laws (4). As discussed in the introduction, given unstable plant dynamics and the discontinuous nature of quantisation, it was not known if the schemes previously proposed could tolerate small differences in encoder and controller initialisation, let alone the perturbation due to occasional bit errors in the channel.

In this paper, we present a preliminary study of, and partial solution to, this problem. We construct a FDEC for the scalar, discrete-time LTI plant (1)-(2) with unbounded process and measurement noise which achieves internal closed loop stability in a mean square sense. I.e. for arbitrary initial internal states $\Psi^{\mathrm{e}}(0), \Psi^{\mathrm{c}}(0)$,

$$
\sup _{t \in \mathbb{Z}_{\geq 0}} \mathrm{E}\left\{|X(t)|^{2}\right\}, \sup _{t \in \mathbb{Z}_{\geq 0}} \mathrm{E}\left\{\left\|\Psi^{\mathrm{e}}\right\|^{2}\right\}, \sup _{t \in \mathbb{Z}_{\geq 0}} \mathrm{E}\left\{\left\|\Psi^{\mathrm{c}}\right\|^{2}\right\}<\infty,
$$

under a suitable condition on the channel bit rate $R$. This rate condition approaches the minimum rate expression of Nair and Evans (2004) in the limit of vanishing initial internal state uncertainty. Furthermore, we will see that the internal states of the encoder and controller reach asymptotic agreement.

\section{STABILISING SCHEME}

In the absence of encoder or controller state uncertainty or structural or memory constraints (apart from causality), it has been established (see, e.g. Tatikonda et al. (2004); Nair et al. (2007)) that if the plant noise is uncorrelated and Gaussian, then the quadratic-optimal encoder-controller consists of three stages: i) a Kalman filter that produces estimates of the plant state from the measurements, given the control inputs, ii) a dynamic quantiser that encodes and transmits the Kalman estimate, according to previously transmitted symbols and iii) a controller which applies the certainty-equivalent LQG-optimal control gain to the received quantised Kalman estimate. Although we assume neither Gaussian nor uncorrelated noise here, and the transmitter here does not know the exact control input, we shall adopt this structure as the basis of our FDEC.

At time $t$, the encoder internal state is 


$$
\Psi^{\mathrm{e}}(t):=\left(\hat{X}(t \mid t-1), X^{\mathrm{e}}(t), L_{G}^{\mathrm{e}}(t), L_{J}^{\mathrm{e}}(t), L_{H}^{\mathrm{e}}(t)\right) \in \mathbb{R}^{2} \times \mathbb{R}_{+}^{3} .
$$

The first component, $\hat{X}(t \mid t-1)$, is a prediction at time $t-1$ of the next plant state, the second component, $X^{\mathrm{e}}(t)$, is the dynamically quantised estimate of the current plant state, and the last three components are dynamic scaling factors used in the quantiser. The first component is updated as

$$
\hat{X}(t+1 \mid t)=a \hat{X}(t \mid t-1)+b k X^{\mathrm{e}}(t)+k^{\mathrm{o}}[Y(t)-C \hat{X}(t \mid t-1)] .
$$

This is essentially a linear Luenberger observer with observer gain $k^{0}$, but with an important difference: due to the uncertainty in the initial decoder internal state, the encoder does not precisely know the control input to be applied at time $t$, but approximates it with its own quantised estimate $X^{\mathrm{e}}(t)$ multiplied by a certainty-equivalent gain $k$.

The second component, $X^{\mathrm{e}}(t)$ is updated according to a predictive, dynamic quantisation scheme, the core of which is the static, $\mu^{v}$-level, non-uniform, symmetric quantiser $q(\cdot)$ of Nair and Evans (2004) (pg. 425). The quantiser points take values in the finite real set

$$
q(x) \in\left\{\Phi(0)<\Phi(1) \cdots<\Phi\left(\mu^{v}-1\right)\right\} \subset \mathbb{R} .
$$

For each $s \in\left[0, \ldots, \mu^{v}-1\right]$ we also define $\kappa(s) \in\left[\kappa_{\min }, \kappa_{\max }\right]$, equal to the half-length of the quantiser interval associated with the point $\Phi(s)$;. for the semi-infinite intervals associated with $s=0$ and $s=\mu^{v}-1, \kappa(s)$ is a nominal half-length. Note that

$$
\kappa_{\min }:=\frac{1}{\left(\mu^{2}-2\right) \mu^{v-2}} \leq \kappa(s) \leq 0.5 \frac{1-\rho^{-1}}{1-\mu^{-1}} \rho^{v}=: \kappa_{\max } .
$$

The encoder proper is based on predictive differential rules with dynamic scaling (see Liberzon (2003) or 'adaptive' delta modulation in Gersho and Gray (1993)). I.e. at time $t$, the symbol transmitted is the unique index $\in\left[0, \ldots, \mu^{v}-1\right]$ of the quantiser point

$$
\varpi(S(t))=q\left(\frac{\hat{X}(t+1 \mid t)-(a+b k) X^{\mathrm{e}}(t)}{L^{\mathrm{e}}(t)}\right) .
$$

The quantised encoder estimate is then updated according to

$$
\begin{aligned}
X^{\mathrm{e}}(t+1) & =(a+b k) X^{\mathrm{e}}(t)+L^{\mathrm{e}}(t) q\left(\frac{\hat{X}(t+1 \mid t)-(a+b k) X^{\mathrm{e}}(t)}{L^{\mathrm{e}}(t)}\right) \\
& =(a+b k) X^{\mathrm{e}}(t)+L^{\mathrm{e}}(t) \varpi(S(t)) .
\end{aligned}
$$

The quantiser scaling factor is generated by the following rules:

$$
\begin{aligned}
L^{\mathrm{e}}(t) & =|a| L_{G}^{\mathrm{e}}(t)+\left|k^{\mathrm{o}} c\right| L_{J}^{\mathrm{e}}(t)+\left|k^{\mathrm{o}}\right| \sigma_{W}, \\
L_{G}^{\mathrm{e}}(t+1) & =L^{\mathrm{e}}(t) \kappa(S(t)) \\
L_{J}^{\mathrm{e}}(t+1) & =\left|a-k^{\mathrm{o}} c\right| L_{J}^{\mathrm{e}}(t)+|b k| L_{H}^{\mathrm{e}}(t)+\sigma_{V}+\left|k^{\mathrm{o}}\right| \sigma_{W} \\
L_{H}^{\mathrm{e}}(t+1) & =|a+b k| L_{H}^{\mathrm{e}}(t)+\eta L^{\mathrm{e}}(t)[1+\kappa(S(t))],
\end{aligned}
$$

where $\sigma_{V}, \sigma_{W}, \eta>0$ are arbitrary constants. These laws differ significantly from Nair and Evans (2004), which had a onedimensional update for the quantiser scaling factor.

At the other end of the channel, the controller simply runs an exact copy of these update laws, on its own internal state

$$
\Psi^{\mathrm{c}}(t):=\left(X^{\mathrm{c}}(t), L_{G}^{\mathrm{c}}(t), L_{J}^{\mathrm{c}}(t), L_{H}^{\mathrm{c}}(t)\right) \in \mathbb{R} \times \mathbb{R}_{+}^{3},
$$

the components of which, however, are not initialised to the same values as the corresponding components of (7). It receives the symbol $S(t-1) \in\left[0, \ldots, \mu^{v}-1\right]$ at time $t \geq 1$ (due to the delay in the channel) and then sets

$$
\begin{aligned}
X^{\mathrm{c}}(t+1) & =(a+b k) X^{\mathrm{c}}(t)+L^{\mathrm{c}}(t) \varpi(S(t)), \\
L^{\mathrm{c}}(t) & =|a| L_{G}^{\mathrm{c}}(t)+\left|k^{\mathrm{o}} c\right| L_{J}^{\mathrm{c}}(t)+\left|k^{\mathrm{o}}\right| \sigma_{W}, \\
L_{G}^{\mathrm{c}}(t+1) & =L^{\mathrm{c}}(t) \kappa(S(t)), \\
L_{J}^{\mathrm{c}}(t+1) & =\left|a-k^{\mathrm{o}} c\right| L_{J}^{\mathrm{c}}(t)+|b k| L_{H}^{\mathrm{c}}(t)+\sigma_{V}+\left|k^{\mathrm{o}}\right| \sigma_{W}, \\
L_{H}^{\mathrm{c}}(t+1) & =|a+b k| L_{H}^{\mathrm{c}}(t)+\eta L^{\mathrm{c}}(t)[1+\kappa(S(t))], \\
U(t) & =k X^{\mathrm{c}}(t) .
\end{aligned}
$$

We remark that the quantities $L_{G}^{x}(t), L_{J}^{x}(t)$ and $L_{H}^{x}(t)(x$ being ' $\mathrm{e}$ ' or ' $\mathrm{c}$ ') can be regarded as the nominal uncertainties associated with the errors

$$
\begin{aligned}
J(t) & :=\hat{X}(t \mid t-1)-X(t), \\
G(t) & :=X^{\mathrm{e}}(t)-\hat{X}(t \mid t-1), \\
H(t) & :=X^{\mathrm{c}}(t)-X^{\mathrm{e}}(t),
\end{aligned}
$$

due to quantisation and noise. As will be seen in the next section, the update rules (12)-(15) and (18)-(20) are designed to closely mimic the dynamics of the corresponding terms (23)(25).

\section{INTERNAL STABILITY ANALYSIS}

Due to the dynamic quantiser in the feedback loop, it turns out to be difficult to obtain recursive bounds directly for mean square states. Instead, we focus on the functional introduced in Nair and Evans (2004),

$$
\|X, L\|_{*}^{2}:=\mathrm{E}\left\{L^{2}+|X|^{2+\varepsilon} L^{-\varepsilon}\right\} \in[0, \infty],
$$

defined for any random variables $X \in \mathbb{R}, L \geq 0$. This has several useful properties: $i$ ) its square root is a pseudo-norm on the space of random variables on $\mathbb{R} \times \mathbb{R}_{+}$; ii) It upper-bounds the mean square values of $X$ and $L$; iii) If the quantiser $q(\cdot)$ with $\mu^{v}$ levels (briefly described in the previous section) is applied to a random variable $X$ with scaling factor $L>0$, then the quantiser error $X-L q(X / L) \equiv X-L \varpi(S)$ and the (nominal) quantiser interval half-length $L \kappa(S)>0$ satisfy

$$
\left\|X-L q\left(\frac{X}{L}\right), L \kappa(S)\right\|_{*} \leq \frac{\zeta}{\mu^{v}}\|X, L\|_{*}, \forall v \in[2,3, \ldots),
$$

where $\zeta>0$ is a constant that depends on $\varepsilon$, the quantiser base $\mu$ and another quantiser parameter, but is independent of $v$ and the distribution of $X, L$.

\subsection{Proof of Stability}

We first establish the mean square stability of the error terms (23)-(25). For any $t \in \mathbb{Z}_{\geq 0}$, let

$$
\begin{aligned}
Z_{G}(t) & :=L_{G}^{\mathrm{c}}(t)-L_{G}^{\mathrm{e}}(t), Z_{J}(t):=L_{J}^{\mathrm{c}}(t)-L_{J}^{\mathrm{e}}(t), \\
Z_{H}(t) & :=L_{H}^{\mathrm{c}}(t)-L_{H}^{\mathrm{e}}(t), \\
F(t) & :=\max \left\{\frac{\left|Z_{G}(t)\right|}{L_{G}^{\mathrm{e}}(t)}, \frac{\left|Z_{J}(t)\right|}{L_{J}^{\mathrm{e}}(t)}, \frac{\left|Z_{H}(t)\right|}{L_{H}^{\mathrm{e}}(t)}\right\}, \\
\alpha(t) & :=\sup _{X(0), \Psi \mathrm{e}(0), \Psi \mathrm{c}(0), V(0), W(0), V(1), W(1), \ldots} F(t) .
\end{aligned}
$$

We have the following result, the proof of which is omitted here:

Lemma 1. Let the FDEC (7)-(22) be used on the plant (1)(2). Then the maximum fractional scaling error (30) decreases monotically to a limiting random variable $F_{*} \geq 0$ with time. 
Similarly, the worst-case maximum fractional error (31) is nonincreasing and, if initially finite, monotonically approaches a constant limit $\alpha_{*} \geq 0$. These results hold whether or not the closed loop is stable.

In the following we assume that $\eta$, a scaling factor parameter of (15), (21), is set equal to $\alpha(0)$. It is straightforward to show that the coupled dynamics of the random errors $\{(G(t), J(t), H(t))\}$ are given by

$$
\begin{aligned}
G(t+1)= & a G(t)+k^{\mathrm{o}} c J(t)+k^{\mathrm{o}} W(t) \\
& -L^{\mathrm{e}}(t) q\left(\frac{a G(t)+k^{\mathrm{o}} c J(t)+k^{\mathrm{o}} W(t)}{L^{\mathrm{e}}(t)}\right) \\
J(t+1)= & \left(a-k^{\mathrm{o}} c\right) J(t)+b k H(t)+V(t)-k^{\mathrm{o} W}(t), \\
H(t+1)= & (a+b k) H(t) \\
& +\left(L^{\mathrm{c}}(t)-L^{\mathrm{e}}(t)\right) q\left(\frac{a G(t)+k^{\mathrm{o}} c J(t)+k^{\mathrm{o}} W(t)}{L^{\mathrm{e}}(t)}(3) 4\right)
\end{aligned}
$$

Applying the pseudo-norm $\|\cdot, \cdot\|_{*}$ to (32), (13) and using the quantiser error bound (27), positive homogeneity and the triangle inequality, we obtain

$\left\|G(t+1), L_{G}^{\mathrm{e}}(t+1)\right\|_{*}$ $\leq \frac{\zeta}{\mu^{\nu}}\left(|a|\left\|G(t), L_{G}^{\mathrm{e}}(t)\right\|_{*}+\left|k^{\mathrm{o}} c\right|\left\|J(t), L_{J}^{\mathrm{e}}(t)\right\|_{*}+\left|k^{\mathrm{o}}\right|\left\|W(t), \sigma_{W}\right\|_{*}\right.$ After dealing in like manner with the $J$ and $H$ terms, we obtain a sublinear, nonnegative recursion

$$
\beta(t+1) \leq \Xi \beta(t)+\chi(t), \forall t \geq t_{\rho},
$$

where

$$
\beta(t):=\left[\left\|G(t), L_{G}^{\mathrm{e}}(t)\right\|_{*}\left\|J(t), L_{J}^{\mathrm{e}}(t)\right\|_{*}\left\|H(t), L_{H}^{\mathrm{e}}(t)\right\|_{*}\right]^{\mathrm{T}},
$$

$$
\begin{aligned}
& \Xi:= \\
& {\left[\begin{array}{lll}
\zeta|a| \mu^{-v} & \left|k^{\mathrm{o}} c\right| & \left|k^{\mathrm{o}}\right| \\
0 & \left|a-k^{\mathrm{o}} c\right| & |b k| \\
\alpha(0)|a|\left(1+\frac{\zeta}{\mu^{v}}\right) & \alpha(0)\left|k^{\mathrm{o}} c\right|\left(1+\frac{\zeta}{\mu^{v}}\right) & |a+b k|
\end{array}\right]}
\end{aligned}
$$

and

$$
\begin{aligned}
\chi(t):= & {\left[\zeta\left|k^{\mathrm{o}}\right| \mu^{-v}\left\|W(t), \sigma_{W}\right\|_{*},\left\|V(t), \sigma_{V}\right\|_{*}+\left|k^{\mathrm{o}}\right|\left\|W(t), \sigma_{W}\right\|_{*},\right.} \\
& \left.\alpha(0)\left(1+\zeta \mu^{-v}\right)\left|k^{\mathrm{o}}\right|\left\|W(t), \sigma_{W}\right\|_{*}\right]^{\mathrm{T}} .
\end{aligned}
$$

Note that $\chi(t)$ is uniformly bounded over time. Thus if $\Xi$ (37) is strictly stable, then the recursion (35) yields uniformly bounded $\beta(t)$. By the upper-bound property of the pseudonorm, $\left\{G(t), J(t), H(t), L_{G}^{\mathrm{e}}(t), L_{J}^{\mathrm{e}}(t), L_{H}^{\mathrm{e}}(t)\right\}$ are then uniformly bounded in mean square. By rewriting the plant dynamics as

$X(t+1)=(a+b k) X(t)+b k[H(t)-G(t)-J(t)]+V(t)$, (39) where $|a+b k|<1$ and $\{V(t)\}$ is uniformly mean-square bounded, it follows from the above that $\{X(t)\}$ is also uniformly mean-square bounded. Definitions (24)-(25), Lemma 1 and the pseudo-norm triangle inequality then imply that so too are all remaining closed loop state variables $\{\hat{X}(t \mid t-$ $\left.1), X^{\mathrm{e}}(t), X^{\mathrm{c}}(t), L_{G}^{\mathrm{c}}(t), L_{J}^{\mathrm{c}}(t), L_{c}^{\mathrm{e}}(t)\right\}$. This conclusion is summarised below:

Theorem 2. Let the finite-dimensional encoder-controller (FDEC) (7)-(22) be used on the partially observed, unstable linear plant (1)-(2) having possibly unbounded process and observation noise with uniformly bounded $(2+\varepsilon)$-th absolute moments. If the controller gain $k$ is selected so that $|a+b k|<1$ and if the eigenvalues of the matrix $\Xi$ (37) lie inside the open unit disc, then the plant state (1) and FDEC internal states (7) and (16) are uniformly mean-square-bounded over time.

This leads to the question of whether there actually exists a nonzero initial fractional scaling error bound $\alpha(0)>0$ that leads to stability for given FDEC parameters. Looking at the equation for the matrix $\Xi(37)$, observe that it becomes upper triangular as $\alpha(0) \rightarrow 0$. Noting that the eigenvalues of a triangular matrix are its diagonal elements and that eigenvalues vary continuously with matrix elements [Horn and Johnson (1985)], we immediately have the following corollary.

Corollary 3. If the number of quantiser levels $\mu^{\nu}>\zeta|a|$ and $|a+b k|,\left|a-k^{0} c\right|<1$, then there exists a positive $\alpha(0)>0$ such that the FDEC (7)-(22) used on the plant (1)-(2) achieves internal mean-square stability of all closed-loop states, for any nonzero initial fractional scaling error (30) less than or equal to $\alpha(0)$.

Though we have focused here on scalar plants, we remark that similar results can be shown to hold with vector-valued plant states.

\subsection{Achieving Minimum Rate}

.The sufficient condition $\mu^{v}>\zeta|a|$ of Corollary 3 is stronger than given by the Data Rate Theorem in Nair and Evans (2004) for stochastic plant state stabilisability due to the presence of the factor $\zeta>1$. However, it is possible to approach the infimum data rate by downsampling the plant, as in Nair and Evans (2004).

Specifically, select a large $\tau \in \mathbb{N}$, divide time up into coding cycles $[j \tau, j \tau+\tau-1], j \in \mathbb{Z}_{\geq 0}$ and sample the plant outputs only at times $t=0, \tau, 2 \tau, \cdots$. The plant equations may then be written $\forall j \in \mathbb{Z}_{\geq 0}$ as

$$
\begin{aligned}
X^{\prime}(j+1) & =a^{\tau} X^{\prime}(j)+b a^{\tau-1} U^{\prime}(j)+V^{\prime}(j), \\
Y^{\prime}(j) & =c X^{\prime}(j)+W^{\prime}(j),
\end{aligned}
$$

where

$$
\begin{aligned}
X^{\prime}(j) & :=X(j \tau), Y^{\prime}(j):=Y(j \tau), W^{\prime}(j):=W(j \tau), \\
U^{\prime}(j) & :=\sum_{r=0}^{\tau-1} a^{-r} U(j \tau+r), V^{\prime}(j):=\sum_{r=0}^{\tau-1} a^{\tau-1-r} V(j \tau+r) .
\end{aligned}
$$

For simplicity, set $U(t)=0$ when $t \neq 0, \tau, 2 \tau, \ldots$, so that $U^{\prime}(j)=U(j \tau)$; this choice is suboptimal in terms of performance, but suffices to ensure stability.

Now apply the FDEC (8)-(22) to the primed plant, with parameters

$$
\begin{gathered}
\left|a^{\tau}+b a^{\tau-1} k^{\prime}\right|<1,\left|a^{\tau}-k^{\mathrm{o}} c\right|<1, \sigma_{V}^{\prime}=\sum_{r=0}^{\tau-1}|a|^{\tau-1-r} \sigma_{V} \\
\sigma_{W}^{\prime}=\sigma_{W}, v=\left\lfloor\frac{\log _{2}\left(\zeta|a|^{\tau}\right)}{\log _{2} \mu}\right\rfloor+1
\end{gathered}
$$

noting that $\zeta$ is a parameter not dependent on $v$. As $0 \leq$ $x-\lfloor x\rfloor<1$, the R.H.S. above is always strictly greater than $\log _{2}\left(\zeta|a|^{\tau}\right) / \log _{2} \mu$ and so all the hypotheses of Corollary 3 are satisfied. Thus the primed system is internally mean square stable at times $t=0, \tau, 2 \tau, \ldots$. Using the linear time invariance 
of the original plant, it is then straightforward to establish that the plant states are mean square stable over all integer times $t$.

Noting that $\mu^{v}$ is the number of quantiser levels, the bit rate of this scheme averaged over each coding cycle is

$$
R=\frac{v \log _{2} \mu}{\tau} \leq \frac{\log _{2}\left(\zeta|a|^{\tau}\right)+\log _{2} \mu}{\tau} \rightarrow \log _{2}|a|,
$$

as $\tau \rightarrow \infty$. Thus $R$ can be brought arbitrarily close to the universal infimum $\log _{2}|a|$ for mean square stabilisability by choosing a sufficiently large $\tau$.

We remark that although internal stability is guaranteed, performance will deteriorate the closer the data rate is brought to the infimum value. This is a universal characteristic of all encodercontrollers; see Nair and Evans (2004); Nair et al. (2007).

\subsection{Asymptotic Agreement between Encoder and Controller}

We now argue briefly that once internal mean square stability has been achieved, the differences (25) and (28)-(29) in encoder and controller internal states should tend to zero in mean square.

Denote the closed loop state by $\Psi(t)=\left(X(t), \Psi^{\mathrm{e}}(t), \Psi^{\mathrm{c}}(t)\right)$ and observe that, with independent initial plant state and noise, $\{\Psi(t)\}$ is a continuous-valued Markovian process. Suppose that the hypotheses of Theorem 2 are satisfied, so that the matrix $\Xi$ in the sublinear recursion (35) is strictly stable. Thus as time $t \rightarrow \infty$, we can find an asymptotic upper bound $B>$ $\|\beta(t)\|_{2}$ which depends only on the parameters in $\Xi$ and an upper bound on the noise term $\chi(t)$; it is independent of the initial vector $\beta(0)$ due to "exponential forgetting". Defining $\Lambda^{\mathrm{e}}(t):=\left[L_{G}^{\mathrm{e}}(t), L_{J}^{\mathrm{e}}(t), L_{H}^{\mathrm{e}}(t)\right]^{\mathrm{T}}$, the pseudo-norm property (ii) then implies that $B^{2}>\limsup _{t \rightarrow \infty} \mathrm{E}\left\{\left\|\Lambda^{\mathrm{e}}(t)\right\|_{2}^{2}\right\}$, where $B^{2}$ is independent of the distribution of $\Psi(0)$, provided that $\mid l_{G}^{\mathrm{c}}(0)-$ $l_{G}^{\mathrm{e}}(0) \mid \leq \alpha(0)$. That is, $\left\{\Lambda^{\mathrm{e}}(t)\right\}$ is stable in mean-square Euclidean norm. From (12-(15), the dynamics of the scaling factors are bounded below as

$$
\Lambda^{\mathrm{e}}(t+1) \geq M(\kappa(S(t))) \Lambda^{\mathrm{e}}(t)+v \in \mathbb{R}_{+}^{3}
$$

where

$$
\begin{aligned}
M(p) & :=\left[\begin{array}{lll}
|a| p & \left|k^{\mathrm{o}} c\right| p & 0 \\
0 & \left|a-k^{\mathrm{o}}\right| & |b k| \\
\eta|a|(1+p) & \eta\left|k^{\mathrm{o}}\right|(a+p) & |a+b k|
\end{array}\right] \\
v & :=\left[\left|k^{\mathrm{o}}\right| \sigma_{W} \kappa_{\min }, \sigma_{V}+\left|k^{\mathrm{o}}\right| \sigma_{W}, \eta\left(1+\kappa_{\min }\right)\left|k^{\mathrm{o}}\right| \sigma_{W}\right]^{\mathrm{T}}(42)
\end{aligned}
$$

Observe that the each component of the forcing term $v$ in the superlinear recursion (40) is strictly positive. By (10) and (8), each $\kappa(S(t))$ is a time-invariant function of the current closed loop state $\Psi(t)$ and furthermore, with i.i.d. noise the transitional probability density function $f_{\Psi(t+1) \mid \Psi(t)}$ is also time-invariant. Under suitable technical conditions from Markov chain theory and the Perron-Frobenius theory for products of random nonnegative matrices, it can then be shown that since the recursion (40) is bounded above in mean square norm by a constant independent of the initial state $\Psi(0)$,

$\mathrm{E}\left\{\|M(\kappa(S(t))) \times \cdots M(\kappa(S(0))) \lambda(0)\|_{2}^{2} \mid \Psi(0)=\psi(0)\right\} \rightarrow 0$,

for any $\psi(0)$ such that $\left|l_{G}^{\mathrm{c}}(0)-l_{G}^{\mathrm{e}}(0)\right| \leq \alpha(0)$. However, from (12)-(15), (180-(21) and (28)-(29), the scaling error vector $Z(t):=\left[Z_{G}(t), Z_{J}(t), Z_{H}(t)\right]^{\mathrm{T}}$ evolves according to

$Z(t+1)=M(\kappa(S(t))) Z(t)=M(\kappa(S(t))) \times \cdots M(\kappa(S(0))) Z(0)$.
From (43), it can then be shown that $Z(t) \rightarrow 0$ in mean square Euclidean norm. Noting that the quantiser $q(\cdot)$ is bounded and that $|a+b k|<1$, it then follows from (34) that $X^{\mathrm{e}}(t)-X^{\mathrm{c}}(t) \rightarrow$ 0 in mean square norm as well. Thus, the FDEC recovers from its initial internal state error to yield encoder and decoder states that agree asymptotically.

Fractional Scaling Error Convergence The mean square convergence to zero of the scaling error vector $Z(t)$ has a corollary which suggests that the FDEC updates (15) and (21) can be simplified without sacrificing stability. Note that $\forall t \geq 1$,

$$
\begin{aligned}
\|Z(t)\|_{2}^{2} & \geq \max \left\{\frac{\left|Z_{G}(t)\right|}{L_{G}(t)} v_{\min }, \frac{\left|Z_{J}(t)\right|}{L_{J}(t)} v_{\min }, \frac{\left|Z_{H}(t)\right|}{L_{H}(t)} v_{\min }\right\}^{2}(44) \\
& \equiv F(t)^{2} v_{\min }^{2} \geq F_{*}^{2} v_{\min }^{2}
\end{aligned}
$$

where $v_{\min }>0$ is the smallest component of (42), the equality in (45) comes from definition (30) and the last inequality is a consequence of Lemma 1. Taking expectations, we then have that $v_{\min }^{2} \mathrm{E}\left\{F_{*}^{2}\right\} \leq \mathrm{E}\left\{\|Z(t)\|_{2}^{2}\right\} \rightarrow 0$. Thus we must have $\mathrm{E}\left\{F_{*}^{2}\right\}=0$, implying that $F_{*}=0$ with probability 1 . That is, almost all realisations of the maximum fractional scaling error $F(t)$ (30) decrease monotonically to zero.

As the role of the FDEC parameter $\eta$ in the update equations (15) and (21) is to bound the maximum fractional scaling error $\mid L^{\mathrm{e}}(t)-L\left(^{\mathrm{c}}(t) \mid / L^{\mathrm{e}}(t)\right.$, this suggests that $\eta$ can be allowed to tend to zero with time and that an arbitrarily large initial internal state errors can be handled without losing stability. Indeed, though we do not provide a rigorous analysis here, the simulation results of section 5 are for a scheme with $\eta=0$ and with a large initial fractional scaling error.

\section{SIMULATION RESULTS}

In this section we present Matlab simulations that illustrate the preceding results for initial internal state errors $\mid l_{G}^{\mathrm{e}}(0)-$ $l_{G}^{\mathrm{c}}(0)|=| x^{\mathrm{e}}(0)-x^{\mathrm{c}}(0) \mid=100$ and also consider the effect of recurring bit errors.

We initialise the internal scaling factors (12),(14),(15) of the encoder to 1 and let $l_{G}^{\mathrm{e}}(0)=101, X^{\mathrm{e}}(0)=100$. On the controller side, we initialise (18)-(21) to 1 and set $X^{\mathrm{c}}(0)=0$. We consider the plant (1)-(2) with $a=1.1, b=c=1$ with independent, Gaussian process and observation noise having mean 0 and variance 1 . The initial plant state is also Gaussian with zero mean and variance 1 . The simulation results illustrated here were done over 100 time steps and with the squared states averaged at each time step over 1000 iterations. We set $\mu=$ $2, v=2, k^{0}=1, k=-1.1$, and $\sigma_{W}=\sigma_{V}=1$. Figures $1-3$ correspond to scenario (1), whereas figure 4 corresponds to scenario (2).

Figures 1 and 2 show that the plant has been successfully stabilised for $\eta=0$ and 0.1 . After initial transients, the average value of the squared plant state $X(t)^{2}$ roughly oscillates around 100 in the former and 325 in the latter. This suggests that system performance is highly affected by this value and that setting $\eta=0$ is in fact optimal. This is due to the fact that the fractional error $F(t)$ (30) monotonically decreases to 0 with time. A more thorough analysis of this will be pursued in the future.

Figures 3 and 4 show that the difference between $l_{G}^{\mathrm{e}}(t)$ and $l_{G}^{\mathrm{c}}(t)$ tends to zero in mean square and proportional senses respectively, supporting the analysis in subsection 4.3. 
Finally, in figure 5 we establish mean-square boundedness of the plant states for a binary symmetric channel with probability of error $p_{e}=0.01$, which in digital communications is typically regarded as high. We do not analyse this here, but the intuitively it is related to the fact that the difference between encoder and controller internal states tends to zero in the absence of bit errors. Although each incorrect channel bit introduces an error into the controller internal state, the difference between the two internal states diminishes to zero. Thus if the time constant characterising the reciprocal rate of this decay is short compared to the average time $1 / p_{e}=100$ between bit errors, then the system has sufficient time to recover from each successive bit error. When $p_{e}$ is increased from 0.01 to 0.03 , simulations (not provided here) indicate that stability is lost; that is, a recovery time of 33.3 is insufficient.

We remark that more thorough analyses of stabilisability over noisy channels such as in Sahai and Mitter (2006); Matveev and Savkin (2007) provided necessary and sufficient conditions that related various notions of channel capacity to the openloop plant dynamics. However, the sufficiency of these conditions was generally proven by using randomly generated channel codes. Although it is doubtful that the finite-dimensional scheme here can achieve channel capacity, it is an explicit construction that does not require any channel coding. A more detailed analysis of its performance with bit errors is left as future work.

\section{REFERENCES}

J. Baillieul. Feedback designs in information-based control. In B. Pasik-Duncan, editor, Stochastic Theory and Control Proceedings of a Workshop held in Lawrence, Kansas, pages 35-57. Springer, Oct 2001.

R. W. Brockett and D. Liberzon. Quantized feedback stabilization of linear systems. IEEE Trans. Autom. Contr., 45(7): 1279-89, 2000.

A. Gersho and R. M. Gray. Vector Quantization and Signal Compression. Kluwer, 1993.

S. Graf and H. Luschgy. Foundations of Quantization for Probability Distributions. Springer, 2000.

R. A. Horn and C. R. Johnson. Matrix Analysis. Cambridge University Press, 1985.

D. Liberzon. On stabilization of linear systems with limited information. IEEE Trans. Autom. Contr., 48(2):304-7, 2003.

A. S. Matveev and A. V. Savkin. An analogue of Shannon information theory for detection and stabilization via noisy discrete communication channels. SIAM Jour. Contr. Optim., 46(4):1323-67, 2007.

G. N. Nair and R. J. Evans. Stabilizability of stochastic linear systems with finite feedback data rates. SIAM Jour. Contr. Optim., 43(2):413-36, July 2004.

G. N. Nair, F. Fagnani, S. Zampieri, and R. J. Evans. Feedback control under data rate constraints: an overview. Proceedings of the IEEE, 95(1):108-37, 2007.

A. Sahai and S. Mitter. The necessity and sufficiency of anytime capacity for stabilization of a linear system over a noisy communication link part 1: scalar systems. IEEE Trans. Info. The., 52(8):3369-95, 2006.

S. Tatikonda and S. Mitter. Control under communication constraints. IEEE Trans. Autom. Contr., 49(7):1056-68, July 2004.

S. Tatikonda, A. Sahai, and S. Mitter. Stochastic linear control over a communication channel. IEEE Trans. Autom. Contr., 49(9):1549-61, Sep. 2004.
W. S. Wong and R. W. Brockett. Systems with finite communication bandwidth constraints II: stabilization with limited information feedback. IEEE Trans. Autom. Contr., 44:1049$53,1999$.

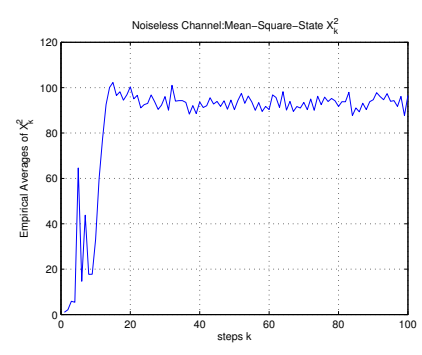

Fig. 1. Noiseless channel, $\eta=0$

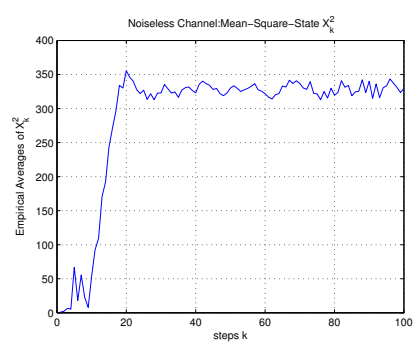

Fig. 2. Noiseless channel, $\eta=0.1$

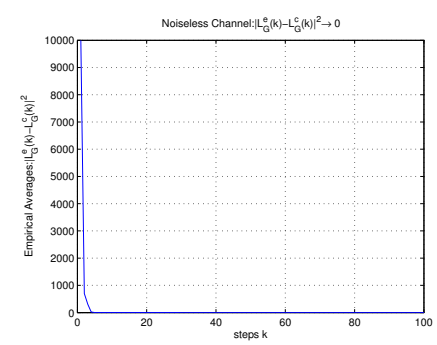

Fig. 3. Noiseless channel, $\eta=0$

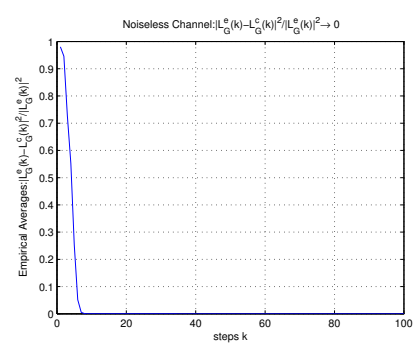

Fig. 4. Noiseless Channel, $\eta=0$

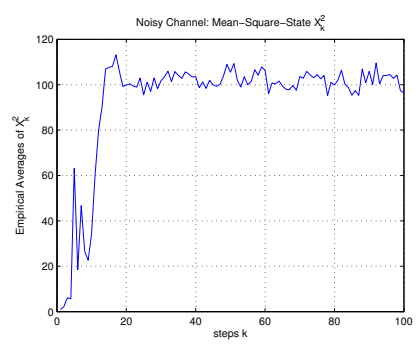

Fig. 5. Binary Symmetric Channel, $p_{e}=0.01, \eta=0$ 